\title{
Eine große Geschichte Gedanken zu einem Roman von Winfried Scharlau
}

\section{von Peter Schreiber}

Es ist ja nicht so selten, daß Mathematiker sich literarisch betätigen. Manche haben es auf diesem Gebiet sogar zu größerem Ruhm gebracht als in der Mathematik. Ich denke dabei z.B. an den Perser Omar Khayyam (1048?-1131?), dessen freizügige Verse im 19. Jh. in Europa bekannt und populär wurden, bevor seine Bedeutung als Algebraiker voll erkannt war, und die auch im 20. Jh. noch mehrfach übersetzt und aufgelegt wurden, oder an Lewis Caroll (alias Charles Lutwidge Dodgson, 1832-1898), über den in diesem Zusammenhang kein weiteres Wort gesagt werden muß. Nicht so bekannt in der ,westlichen Welt", aber dennoch bemerkenswert sind z. B. Helga Königsdorf (geb. 1938), ehemals Professor an der Akademie der Wissenschaften der DDR, der Tscheche Jan Klima mit seinen Kriminalromanen, der sibirische Professor Alexander Wolkow (1891-1977), dessen Kinderbücher rund um den schlauen Urfin, den eisernen Holzfäller und den Scheuch sich inzwischen auch den westdeutschen Buchhandel erobert haben, und die sowjetische Mathematikerin Jelena S. Wentzel (1907?), die unter dem Pseudonym I. Grekowa (d. h. Frau Y) Romane und Erzählungen schrieb. Als ihr spektakulär kritischer Roman „Der Lehrstuhl“ (1978) 1981 in der DDR in deutscher Übersetzung erschien, wurde ich von einer führenden Zeitung um eine Besprechung gebeten. Auf höchste Intervention wurde sie nicht gedruckt - und ich bekam ein ungewöhnlich hohes Honorar. Ich könnte diese Liste endlos fortsetzen mit Hinweisen auf literarische Betätigung von Johannes Kepler, Bachet de Méziriac, Sofia Kowalewskaja, auf die vielen, die Mathematik nur studiert oder ,anstudiert" haben, um dann erfolgreiche Berufsschriftsteller zu werden, und die zahlreichen Autobiographien namhafter Mathematiker, aber das ist hier nicht das Thema.
Jedesmal, wenn sich wieder ein Mathematiker in die stattliche Schar der Dichter und Schriftsteller eingereiht hat, werden den Fachkollegen vor allem zwei Fragen interessieren: 1. Künstlerische Betätigung von Mathematikern (auch auf dem Gebiet der Musik oder Malerei) kann, wenn sie als solche bekannt wird, dazu beitragen, das schlechte Image des Mathematikers in der Öffentlichkeit als eines trockenen, pedantischen, weltabgewandten Sonderlings ein wenig zu korrigieren. Taugt also das neue Werk in diesem Sinne etwas, und wird es hinreichend deutlich, daß ein Mathematiker es vollbracht hat? 2. Was erfährt der Leser gegebenenfalls über Mathematik bzw. über das Leben und die Arbeit des Mathematikers? (Wenn Frage 1 positiv zu beantworten ist, macht es natürlich nichts, wenn im literarischen Werk gar nichts über Mathematik steht.)

Was ist aus dieser Perspektive über den Roman „I megalli istoria“ (Die große Geschichte) des Ex-DMVPräsidenten Winfried Scharlau zu sagen? Zum ersten: Alle, die ihn gelesen haben und die ich darüber befragte, waren begeistert. Leider sind es vorläufig wohl fast nur Kollegen, denn Herr Scharlau fand bisher keinen Verlag für sein Buch und hat es daher zunächst im Selbstverlag herausgegeben. Es ist aber wirklich Literatur. Herr Scharlau gehört nicht nur zu den wenigen noch Lebenden, die jeden angefangenen Satz grammatisch und stilistisch richtig beenden können. Sein Buch ist voller Poesie, voller herrlicher und sprachgewaltiger Schilderungen von Menschen, Landschaften und Stimmungen. Natürlich hat es auch eine Handlung, aber die ist beinahe Nebensache angesichts von soviel sprachlicher Schönheit. Offensichtlich trägt es teilweise autobiographische Züge. Der „Held“, ein Mathematikprofessor namens Christopher 
Faust an einer norddeutschen Universität, reist beständig zwischen dieser nördlichen Provinzstadt und seiner geliebten griechischen Inselwelt hin und her. In der letzteren spielt sich der größere Teil der Geschichte ab. Der andere Teil brachte zumindest mir vertiefte Einsicht in den bestürzenden Grad innerer Verrottung des bundesdeutschen und vielleicht überhaupt des Hochschulwesens westlicher Prägung. Das bedarf keiner vordergründigen Schilderung, man muß es fast zwischen den Zeilen lesen, und dennoch ist es, nachdem ich alles oben Beschriebene und auch die herrlich natürlichen, unbefangenen Schilderungen des Sexuallebens des Helden gebührend genossen habe, der bleibende Eindruck: Das quälende und dennoch oft vergebliche Streben nach wissenschaftlicher Leistung, aber nicht mehr um der Wahrheit willen oder um irgend einer Menschheit zu dienen, sondern einzig um der Karriere willen, die schludrige Dienstauffassung, wenn die Spitze der Karriereleiter erklommen ist, die traurige Symbiose zwischen solcher Art von Hochschullehrern und der staatlichen Hochschulbürokratie. Eine mißlungene mathematische Arbeit landet in einem Pariser Altpapiercontainer. Faust flüchtet aus dieser für ihn unbefriedigenden Arbeitswelt ins Private, in die Natur, in die Gesellschaft der in einer ganz anderen Welt lebenden griechischen Inselbewohner: Fischer, Hirten, mehr oder weniger windige Geschäftsleute. Jeder Grieche, so habe ich gelernt, ist ein wenig Odysseus: Er tendiert dazu, sich in der Ferne herumzutreiben und seine Familie jahrelang zu vernachlässigen. Die Griechin revanchiert sich, indem sie ihren Mann betrügt. Und dennoch verbinden alle diese Menschen auf eine unnachahmliche Weise ein schlichtes, naturnahes Leben mit dem Nachdenken über Prinzipielles. Ich verstehe jetzt besser, wieso gerade dieses Volk einen Aristoteles, einen Euklid und einen Apollonios hervorbringen konnte. Gelegentlich ist auch von richtiger Mathematik die Rede und dann immer so, daß es meiner Ansicht nach auch ein Abiturient verstehen kann. Köstlich zum Beispiel die Szene, in der die griechischen Männer sich vergeblich bemühen, die Größe eines vermessenen Grundstückes zu überprüfen, und der Landmesser einsehen muß, daß er die gelernte und seit Jahrzehnten praktizierte Methode nicht versteht. Bemerkenswert auch die Diskussion zwischen Faust und seiner Eva über partielle Integration oder über die Heisenbergsche Unschärferelation.

Frage 1 ist also positiv zu beantworten, Frage 2 ist schwierig. Das Buch macht sehr deutlich, daß Mathematiker „richtige Menschen“ sind. Es weckt Lust, die griechische Inselwelt kennenzulernen. (Das wird der Rezensent im Sommer 2000 tun.) Es weckt Lust, eine Affäre mit einem ganz jungen Mädchen anzufangen. (Das wird der Rezensent nicht tun, denn er hat es schon vor 28 Jahren getan und ist seitdem glücklich mit dem immer noch jungen Mädchen verheiratet.) Lust, ein Mathematiker oder gar ein Hochschullehrer zu werden, weckt es leider nicht.

P.S.: Die große Geschichte (382 Seiten) kann für $25,-\mathrm{DM}+$ Versandkosten nur direkt beim Autor (scharla@math.uni-muenster.de bzw. Ignatiusstr. 35, 48329 Havixbeck) bezogen werden.

\section{Adresse des Autor}

Prof. Dr. Peter Schreiber

Institut für Mathematik und Informatik

Ernst-Moritz-Arndt-Universität

17487 Greifswald

schreibe@mail.uni-greifswald.de

\section{mathemas www.ordinate.de}

× 043132 888-10/凶-12, Email: info@ordinate.de $\rightarrow$ Software for mathematical people ! Mathematica ${ }^{\mathrm{TM}}$, MathType $^{\mathrm{TM}}$, Extend, Geometrix, KaleidaGraph, MathSHelp, MathWare ${ }^{\circledR}$, Microsoft, Palisade, StatView und a.m.

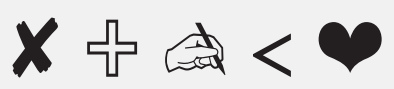
mathemas, Dipl. Math.Carsten Herrmann, M. Sc. Mehr als 15 Jahre Erfahrung mit Software-Distribution !
Königsbergerstr. 97, 24161 Altenholz 